\section{Estudio seminal revela mecanismos celulares de la degeneración hepática grasa de origen no alcohólico}

Una de las posibles consecuencias adversas de la obesidad es el llamado "síndrome metabólico", que se caracteriza por resistencia a la insulina, hipertensión arterial y dislipemias, problemas que pueden llevar a un peligroso deterioro de la salud. Este síndrome se asocia, a su vez, con una afección cada vez más común que actualmente se conoce en español por el nombre de "degeneración hepática grasa de origen no alcohólico" [NAFDL, por el inglés nonalcoholic fatty liver disease]. La enfermedad, que comienza con la acumulación de grasa en el parénquima hepático en pacientes sin antecedentes de alcoholismo, puede a la larga acompañarse de una reacción inflamatoria y degenerar en fibrosis parenquimatosa e incluso cirrosis hepática. Hasta este momento, las causas de la NAFDL, que es una forma de lipotoxicidad, no se habían descifrado del todo, aunque se sabía, por ejemplo, que algún papel desempeñaba el factor de necrosis tumoral alfa (FNT- $\alpha$ ) y que los ácidos grasos libres (AGL) tenían un efecto tóxico directo sobre los hepatocitos y llevaban a la acumulación de grasa intrahepática.

La obesidad también desempeña un papel clave en la enfermedad. Las personas obesas tienen aumentada la cantidad de AGL que penetra en el hepatocito, y si también tienen resistencia a la insulina, el hígado produce más AGL de los que se pueden metabolizar y eliminar, con el resultado de que los AGL se acumulan en el parénquima hepático. En personas con NAFDL, la concentración plasmática de ácidos grasos también suele estar elevada y se correlaciona con el grado de afectación hepática.

Feldstein et al., de la Clínica Mayo en Rochester, Minnesota, Estados Unidos de América, acaban de publicar los resultados del primer estudio que define los mecanismos celulares de la lipotoxicidad hepática mediada por los AGL. El estudio se llevó a cabo en distintas fases. En una fase in vitro, hepatocitos cultivados de distintos tipos de ratones se incubaron 24 horas con una mezcla de AGL. Al microscopio, la inmunofluorescencia reveló cantidades abundantes de catepsina B (ctsb) en el citosol de más de $80 \%$ de las células. Esta sustancia normalmente se encuentra dentro de los lisosomas y su presencia en el citosol es un claro indicador de permeabilidad lisosómica. Los investigadores también examinaron por el mismo método cultivos celulares que no fueron incubados con AGL y observaron que la ctsb estaba concentrada dentro de los lisosomas. En un siguiente paso incubaron algunas células con AGL únicamente, y otras con AGL más un fármaco inhibidor de ctsb (R-3032). La incubación redujo notablemente la cantidad de CTSB en el citosol celular, confirmándose así la función mediadora de la ctsb en la permeabilidad lisosómica asociada con los AGL.

Con objeto de averiguar si los AGL estimulan la expresión del FNT- $\alpha$, los investigadores midieron la cantidad de ARN mensajero de este factor en los hepatocitos y observaron un aumento de más de 100 veces en los que fueron incubados con AGL con respecto a los que no lo fueron. También determinaron que la expresión del FNT- $\alpha$ está a su vez mediada por activación del factor nuclear $\kappa B(F N-\kappa B)$.

Los resultados anteriores se confirmaron en seres humanos con NAFDL. Muestras de hígado de pacientes con esta afección y de personas sin enfermedad hepática obtenidas por biopsia se examinaron mediante inmunofluorescencia. Nuevamente se observó que en las personas sanas la ctsb estaba dentro de los lisosomas hepatocíticos, mientras que en las personas con NAFDL, la ctsb se encontraba dispersa en el citosol celular. Además, la cantidad de ctsb mostró una correlación directa con la gravedad de la afectación hepática, de tal manera que las mayores concentraciones se detectaron en pacientes con esteatohepatitis avanzada.

Por último, los investigadores se propusieron determinar si la inactivación de la ctsb podía proteger contra la NAFDL de origen alimentario. Con ese fin pusieron a ratones salvajes normales y a ratones con inactivación de la ctsb (ratones $\operatorname{ctsb}^{-1-}$ ) a comer alimentos con un alto contenido de carbohidratos durante 16 semanas, al cabo de las cuales ambos grupos de ratones se volvieron muy obesos. También alimentaron a otro grupo de ratones 16 semanas con una dieta estándar para roedores y en ese grupo no se produjo obesidad. Los hígados de estos últimos ratones y de los ratones $c t s b^{-1}$ obesos tenían un aspecto macroscópico normal y no se produjo hepatomegalia; al microscopio, no había indicios de esteatosis macrovesicular. En cambio, los hígados de los ratones salvajes obesos pesaban mucho más que los de los otros animales y el examen microscópico reveló una esteatosis macrovesicular difusa y avanzada. Cuando a algunos de los 
ratones salvajes obesos se les administró R-3032 durante los últimos 7 días de la dieta a base de carbohidratos, la esteatosis hepática desapareció por completo y las concentraciones séricas de alaninoaminotransferasa, que es un marcador de lesión hepática, habían disminuido.

En los ratones salvajes obesos se observó el mismo síndrome dismetabólico que afecta a los seres humanos con NAFDL: concentraciones séricas elevadas de AGL, insulina, leptina y FNT- $\alpha$. Este síndrome no se observó en los ratones $c t s b^{-1-}$ ni tampoco en los ratones salvajes que fueron tratados con R-3032. Cabe deducir que la desactivación de la ctsb por mecanismos farmacológicos o genéticos protege contra la esteatosis hepática y el síndrome dismetabólico que se asocia con ella. En los ratones salvajes obesos también se observó que la expresión de ARN de FNT- $\alpha$ era ocho veces mayor que en animales delgados y en ratones $c t s b^{-1-}$ obesos. No obstante, el tratamiento con R-3032 suprimió por completo este aumento, lo cual confirma que el FNT- $\alpha$ interviene en la esteatosis hepática de origen alimentario. Esto se verificó, además, en ratones con inactivación del FNT- $\alpha$ (ratones FNTR1 ${ }^{-1-}$ ) que fueron sometidos a la alimentación con alto contenido de carbohidratos. Aunque estos ratones se hicieron obesos, ninguno de ellos tuvo esteatosis hepática ni síndrome metabólico.

Gracias a este estudio seminal, ahora se entienden los mecanismos celulares mediante los cuales los AGL lesionan el parénquima hepático en personas con obesidad. Cuando hay concentraciones muy elevadas de lípidos en la circulación, las células del organismo donde estos normalmente se almacenan no dan abasto y los lípidos se quedan flotando libremente en forma de AGL en el citosol celular. En los hepatocitos, esto provoca la cadena de reacciones lipotóxicas observadas en la NAFDL. Esta información es trascendental, puesto que constituye la base para la elaboración futura de fármacos que interrumpan la cadena de reacciones nocivas. En la actualidad, no se dispone de ningún tratamiento para la NAFDL más allá del ejercicio y una sana alimentación. (Feldstein AE et al. Free fatty acids promote hepatic lipotoxicity by stimulating TNF- $\alpha$ expression via a lysosomal pathway. Hepatology. 2004;40(1):185-194.)

\section{La adversidad económica y su asociación con la mortalidad de menores de un año en México}

Durante el período de 1986 a 1996, México, al igual que otros países de América Latina, sufrió una crisis económica que acentuó la pobreza y la desigualdad. Estos reveses no han desaparecido del todo, pese a que en la década de los noventa se pro- dujo una mejoría económica ostensible. Es apenas ahora que las consecuencias sociales y sanitarias de esta situación se empiezan a documentar, sobre todo los efectos que han tenido sobre la salud y la mortalidad la reducción del estándar de vida y del acceso a las necesidades básicas, a la atención sanitaria y a los programas de asistencia social.

Los factores citados suelen ejercer el efecto más marcado en los niños menores de un año debido a su poca resistencia física. El Fondo de las Naciones Unidas para la Infancia (UNICEF) fue una de las primeras organizaciones en advertir un aumento de la mortalidad de menores de un año (mortalidad infantil) en América Latina después de la adopción de los programas de ajuste estructural encaminados a combatir los efectos de la crisis económica. Dichos programas llevaron a un descenso del salario real y a un recorte de programas asistenciales que amenazaban con truncar el descenso de la mortalidad infantil que se había logrado antes de la crisis.

Curiosamente, sin embargo, los estudios no han confirmado la presencia en los últimos años de un revés en el descenso de la mortalidad infantil en general. En América Latina, la mortalidad infantil ha seguido bajando hasta fines del siglo pasado. Lo que aún no se ha determinado es si la crisis económica y los programas de ajuste estructural han tenido un efecto selectivo sobre la mortalidad infantil en los sectores más vulnerables de la población que no se refleja en las tasas de mortalidad generales.

En México, uno de los países más sacudidos por la crisis y los programas de ajuste, un grupo de investigadores ha explorado esta posibilidad mediante un estudio basado en datos de natalidad (obtenidos de la Encuesta Nacional de la Dinámica Demográfica para 1986-1991 y 1992-1996) encaminado a detectar diferencias en la mortalidad observada en los diferentes estratos sociales, definidos en términos de ciertas variables, tales como escolaridad materna, paridad, participación del jefe de familia en la fuerza de trabajo, infrasestructura doméstica (abastecimiento de agua, corriente eléctrica, tipo de piso, etc.), lugar de residencia (urbano, rural). Los investigadores emplearon un modelo de regresión logística para analizar la mortalidad infantil, según el período en que se produjo la muerte (período neonatal: $<28$ días de nacido; período posneonatal: 28 días de nacido o más).

Los resultados revelaron que se produjo un descenso de la mortalidad infantil general de 36,3 por 1000 nacidos vivos en el período de 1986-1991, a 27, 9 por 1000 nacidos vivos en el de 1992-1996. Este descenso se observó predominantemente en las muertes del período posnatal; la mortalidad en el período neonatal descendió muy levemente de un período a otro. Por otra parte, el análisis de re- 